\title{
Laser-Welded Steel Foils with Sapphire Substrates
}

\author{
Araceli de Pablos-Martín ${ }^{1} \cdot$ Christian Grosse $^{1} \cdot$ Andreas Cismak $^{1} \cdot$ Thomas Höche $^{1}$
}

Received: 30 December 2015/Revised: 7 March 2016/Published online: 4 May 2016

(C) The Chinese Society for Metals and Springer-Verlag Berlin Heidelberg 2016

\begin{abstract}
A nanosecond pulsed laser was used to weld stainless steel foils of $10 \mu \mathrm{m}$ thickness to the sapphire substrates. The microstructure of the bonded interface was studied. Both materials were partially ablated under the influence of the laser beam. An inhomogeneous distribution of the steel and sapphire along the bonded interface is observed. The electrical resistance of the steel foil was measured before and after laser welding, showing that the weld slightly increases the electrical resistance but still keeps the values acceptable for electrical contacts.
\end{abstract}

KEY WORDS: Laser welding; Stainless steel; Sapphire; Electrical resistance

\section{Introduction}

Materials for microelectromechanical systems (MEMS) undergo rapid development, covering many properties and applications [1]. In that sense, stainless steel is a common material applied in different industries, with a wide application potential, due to its chemical resistance, high mechanical strength, good heat and electric conductivity, low cost and of course corrosion resistance. It achieves its stainless characteristics through the formation of an invisible and adherent chromium-rich oxide film. It is hence not surprising that it has also been considered for applications in the MEMS field. Some of the recent advances in the use of stainless steel in MEMS include: packaging [2], substrates for biocompatible devices, like pressure sensors [3], substrates in microfluidic devices [4] and substrates for

Available online at http://link.springer.com/journal/40195

Araceli de Pablos-Martín

pablos-martin@imws.fraunhofer.de

1 Fraunhofer Institute for Microstructure of Materials and Systems IMWS, Walter-Hülse-Straße 1, 06120 Halle, Germany electromechanical transducer materials for sensors and actuators as an alternative to the most common $\mathrm{Si}$ substrates, offering high fracture toughness even for a plates less than $50 \mu \mathrm{m}$ thickness [5]. Even in the fields of organic light-emitting diodes (OLEDs) [6, 7] and photovoltaics [8], stainless steel has been used as substrate. One of the advantages of the use of metallic substrates for OLEDs instead of glass is a higher thermal conductivity in metals, which facilitates the heat dispersion from the device, constituting the metal top organic light emission diodes (MTOLEDs) [7].

On the other hand, sapphire possesses high hardness, high mechanical stability, chemical durability and heat resistance, presents a high ablation threshold for laser micromachining and exhibits optical transparency over a large spectral range [9]. Thus, sapphire is widely used in the field of optical components and micromechanical devices [10, 11]. Its applications include windows for high-pressure optical cells and pressure devices [12]. Sapphire has been bonded to other materials like metals [13], including gold and silver [14], and alloys Inconel ${ }^{\circledR}$ through a $\mathrm{Cu} / \mathrm{Ni}$ interlayer [12]. In our previous works, two sapphire substrates were laser-welded through a 50-nm titanium thin film [15], as well as through glass thin films [16-18]. 
Consequently, welding of sapphire to steel may have a great potential within the MEMS field. One of the possible applications would be the production of electrically conducting steel structures on sapphire substrates. Laser welding of the stainless steel with transparent materials, like polymers [19] and sapphire, has been reported only in a few works [20, 21]. In the latter references, a picosecond pulsed laser and a continuous-wave laser were used, respectively. The effect of laser irradiation on stainless steel plate and the subsequent formation of nanoparticles were reported [21].

In the present paper, the laser welding process of a sapphire substrate and a stainless steel foil through the irradiation with a nanosecond pulsed laser is discussed, which, to the best of our knowledge, was not reported until now. The structural characterization of the bond interface, as well as electrical measurements of the stainless steel bonded with the sapphire, have been also carried out. The electrical resistance values were compared to that of nonprocessed stainless steel, in order to study the influence of the bonded area.

\section{Materials and Methods}

\subsection{Laser Welding}

According to the setup shown in Fig. 1, a stainless steel (Goodfellow GmbH, Germany) stripe of $10 \mathrm{~mm}$ length, $800 \mu \mathrm{m}$ width and $10 \mu \mathrm{m}$ thickness was placed over a $10 \mathrm{~mm} \times 10 \mathrm{~mm}$ c-sapphire substrate polished on both sides. Prior to welding, the stainless steel foils and the sapphire substrates were thoroughly cleaned in acetone and ethanol in an ultrasonic cleaner. A second $10 \mathrm{~mm} \times 10 \mathrm{~mm}$ sapphire substrate was then placed over the stainless steel foil, forming a sandwich (Fig. 1a). The sample was placed in a custom-made sample holder, which consists in two 4-mmthick square-shaped steel plates with a round opening of $3 \mathrm{~mm}$ diameter. The sandwich sample is placed between

\section{(a)}

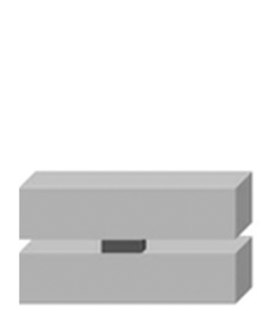

$$
\text { (b) }
$$

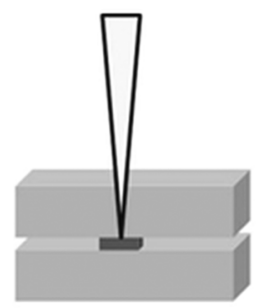

Fig. 1 Schematic of setup. a Sample consisting in two sapphire substrates (light gray) and the stainless steel foil (dark gray). b The laser beam is focused at the upper sapphire/stainless steel interface. c At the end of the laser process, the bottom sapphire is easily removed and only the upper sapphire remains bonded with the steel foil (for clarity, the sample holder is not shown) both plates in the middle of the opening, through which the laser beam is guided to hit the sample. Four screws placed in each corner of the plates are used to put pressure on the sample and bring the sandwich constituents in close proximity. The irradiation source was a nanosecond pulsed Nd:YAG laser (Xiton Photonics) implemented into a microSTRUCT C laser micromachining workstation (3DMicromac AG), operated at $532 \mathrm{~nm}$. A galvanometer scanner unit was employed, and the laser beam moves over the workpiece that remains fixed. A focal length of the objective amounting to $255 \mathrm{~mm}$ was employed. The laser parameters were optimized to: $5 \mathrm{kHz}$ repetition rate, $25.5 \mathrm{~J} / \mathrm{cm}^{2}$ laser fluence on the sample and scan speed of $5 \mathrm{~mm} / \mathrm{s}$, which results in $95.6 \%$ pulse overlap.

The laser beam was focused precisely at the interface between the upper sapphire substrate and the stainless steel foil (Fig. 1b). The lower sapphire substrate acts as structural stabilizer of the entire package. After the laser welding process, the bottom sapphire is easily removed and only the upper sapphire becomes bonded with the steel foil (Fig. 1c).

Single lines transversal to the steel stripes length were irradiated with the laser. Under the same conditions, 20 lines separated by $50 \mu \mathrm{m}$ between them were irradiated in order to have enough bonded area to prepare a cross-sectional sample and investigate the structure and composition of the bonded interface.

\subsection{Light Microscopy}

The microstructure of the welded regions was evaluated by an optical microscope Leica DM RXE-650H.

\subsection{Structural Characterization by Scanning Electron Microscopy (SEM) and Energy- Dispersive X-ray Spectroscopy (EDXS)}

Planar and cross-sectional samples of the bonded specimen through several irradiated lines separated by $50 \mu \mathrm{m}$ were prepared using an argon ion beam (Gatan Ilion $\left.{ }^{+\mathrm{TM}} \mathrm{II}\right)$. The prepared cross section of the bonded stainless steel/sapphire was studied by SEM using a Carl Zeiss Supra 55VP microscope. The secondary electron (SE) detector as well as the angle-selective backscattered electron (AsB) detector were employed. EDXS analyses of $\mathrm{Al}$ ( $K$-line, $1560 \mathrm{eV}), \mathrm{Fe}(K$-line, $7112 \mathrm{eV})$ and $\mathrm{Cr}(K$-line, $5989 \mathrm{eV})$ were recorded at $20 \mathrm{kV}$ in the same SEM microscope.

\subsection{Electrical Measurements}

The electrical resistances of the stainless steel stripes bonded to the sapphire were measured according to a fourpoint probe configuration. Four microneedles of $1-\mu \mathrm{m}$ tip 
radius were each positioned with a micromanipulator Suss PH-150 aligned over the stripe. A current is passed through the two outer needles, while the voltage is measured between the two inner needles. The distance between the needles was $1 \mathrm{~mm}$ from the outer to the inner needle and $3 \mathrm{~mm}$ between the inner needles. For the electrical measurements, a parameter analyzer Keithley 4200-SCS was used. For measuring the I-V curve, a current sweep from -500 to $+500 \mu \mathrm{A}$ was applied. The step size of the sweep was $5 \mu \mathrm{A}$, and a compliance of $200 \mathrm{mV}$ was set. The current and voltage were measured, and the corresponding resistance was calculated automatically by the slope of the I-V curve from a linear regression fit, which shows in every case an ohmic behavior. A total of four stripes were measured, and three measurements were taken in each stripe.

For comparison, the electrical resistances of two stainless steel stripes of the same dimensions without any laser irradiation were also measured under the same conditions.

\section{Results and Discussion}

According to Bergström et al. [22], the optical absorption of the stainless steel at $532 \mathrm{~nm}$ is between 44 and $65 \%$. The reflectance was also reported, giving values between 35 and $54 \%$. Both magnitudes depend on the surface oxides, surface roughness and alloying elements. The absorbance is higher for the green light compared to the infrared. In our setup, the applied 532-nm laser light goes through the upper sapphire substrate, which transmits approximately $86 \%$ of the incident light at $532 \mathrm{~nm}$, and it is partially absorbed by the stainless steel foil.

The effect of ns laser pulses at interfaces must be taken into account [23]. Laser absorption greatly increases when the laser beam is incident from the material of higher refractive index to that of the lower refractive index, because of the creation of constructive interferences of laser light [23]. According to our setup, between the bare sapphire $(n=1.75)$ and the stainless steel there is a small air gap $(n=1)$. Greenaway et al. [24] reported an expression to quantify the increase in the local incident electric field by the generated constructive interferences. For sapphire, an increase in the laser intensity by as much as $62 \%$ was calculated. This phenomenon leads to heat accumulation on the interface, inducing ablation of the sapphire and stainless steel, which melt by heat conduction.

After the laser welding, both materials are joined and the external surface of the sapphire is free of damages. Figure 2 displays top-view optical micrographs of the bonded interface between the sapphire and steel. The irradiated single lines are perfectly observed. Debris of the ablated material are discerned on both sides of the irradiated lines
(Fig. 2a), as well as at the edges of the steel foil (Fig. 2b). The debris are consequence of the microexplosions generated by the laser plume and shock wave, which lead to the ablation of both materials. The weld seam presents a thinner inner seam (Fig. 2a). The outer region represents the width of the modified sapphire, while the inner region indicates the width of the modified stainless steel, similar to that reported in Ref. [20] for glass-to-aluminum welds. This double seam was confirmed by the cross-sectional inspection below.

From the steel foil side, the molten material is observed forming a wave-like microstructure (Fig. 3). The width of the laser-affected zone on the steel side is $24 \mu \mathrm{m}$. The cross-sectional optical micrograph of the welded sample in which 20 lines separated by $50 \mu \mathrm{m}$ were irradiated is displayed in Fig. 4. Between the sapphire and steel, a gap of 6-8 $\mu \mathrm{m}$ wide is observed. This gap was filled with the ablated material from both steel and sapphire. The steel foil presents bumps in those positions which were irradiated, with the hillock pointing in the direction of the laser entry (from sapphire side). Similar bumps were also observed in our previous works, in which the sapphire substrates were laser-welded through different glass thin-film solders [16, 18]. In these works, the upper sapphire substrate was ablated because of laser irradiation and molten solder material filled the ablated region, forming similar bumps and leading to a solder material containing material from the upper substrate. This is the case shown in Fig. 4, in which it is clear that the laser irradiation ablates the sapphire and the molten material occupies the ablated area.

There are no cracks in the ablated region, differently to that reported in Ref. [20] also for sapphire/stainless steel joints. The generated heat is not enough to bond the lower sapphire located in the setup as stabilizator (Fig. 1), and hence, it can be easily removed after laser welding.

To go deeper into microstructure analysis, this cross section was studied by SEM and EDXS analysis. Figure $5 \mathrm{a}, \mathrm{b}$ displays the cross section of one of the observed bumps. The gap between the sapphire substrate and the steel foil is clearly observed. Round-shaped voids are found to be distributed along the whole re-solidified material in the interface (Fig. 5b). In Ref. [21], it was reported that liquid drops of the stainless steel with a size in the lower nanometer range can be formed in the vapor plume, leading to nanoparticles after the cooling.

EDXS elemental analyses were performed in different regions of the bump shown in Fig. 6. Table 1 displays the results. According to the stainless steel general composition [25], only $\mathrm{Fe}$ and $\mathrm{Cr}$ were analyzed to simplify the analysis.

EDXS localized analyses indicate the mixing of both materials and an inhomogeneous distribution of the steel and the sapphire in the bump interface, since different $\mathrm{Fe} /$ 

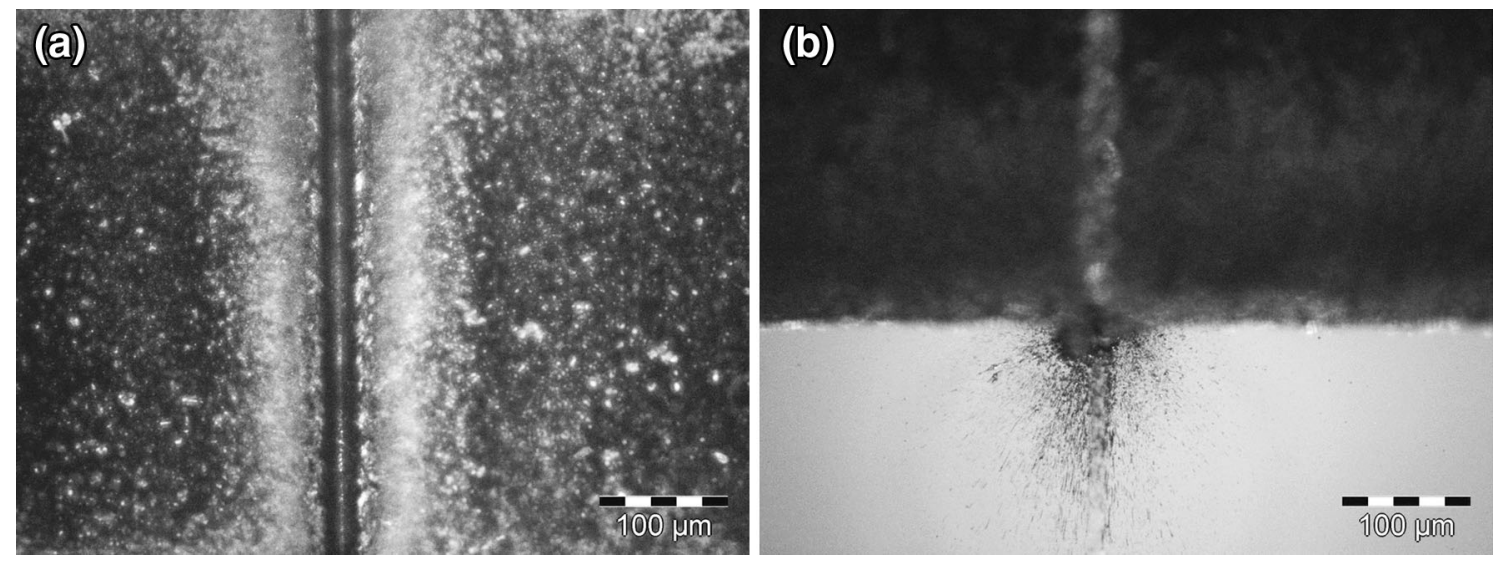

Fig. 2 Top-view optical micrographs of the sapphire/steel interface in the middle of the irradiated line a and showing one of the extremes of the line b. Sapphire substrate is upward

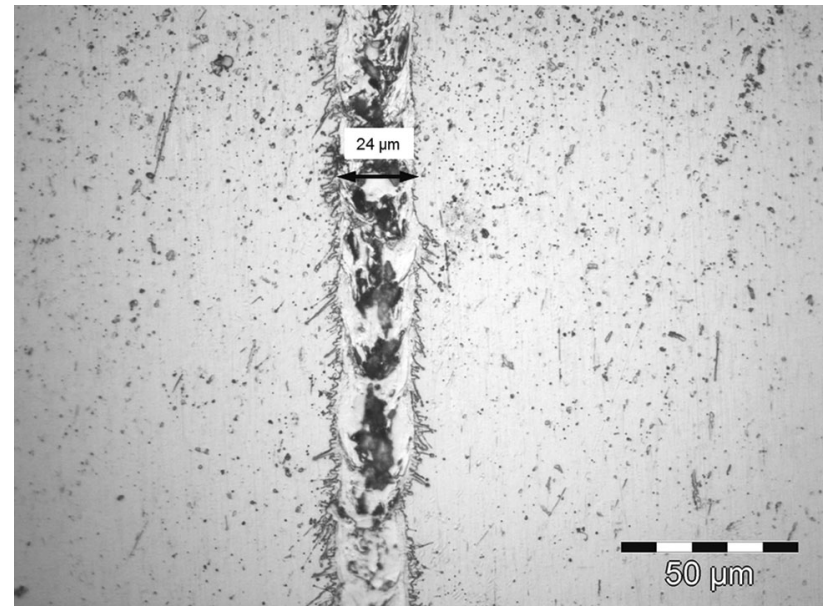

Fig. 3 Top-view optical micrograph of the irradiated line with the stainless steel foil upward

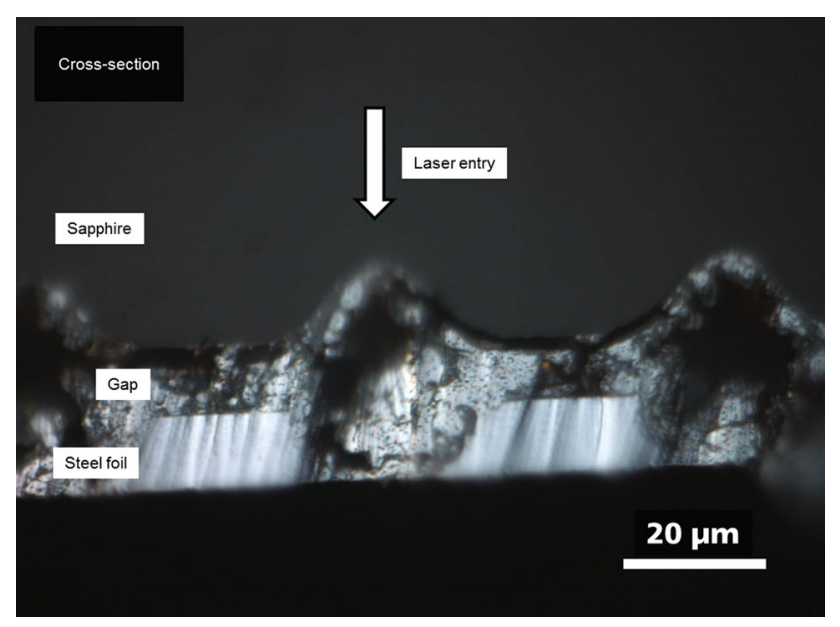

Fig. 4 Optical micrograph of the cross section of the stainless steel/ sapphire interface
$\mathrm{Al}$ ratios are observed in the interface formed. The $\mathrm{Fe} / \mathrm{Cr}$ ratio of the stainless steel foil is around 4 and remains nearly constant in all the measured areas in Table 1 . Thus, a decomposition of the steel is unlikely to take place. Reactions between stainless steel substrates coated by ion beam sputtering with $\mathrm{Al}_{2} \mathrm{O}_{3}$ were reported in Ref. [26], showing the formation of $\mathrm{Fe}-\mathrm{O}-\mathrm{Al}$ and $\mathrm{Cr}-\mathrm{O}-\mathrm{Al}$ species.

This intermixing of both materials and their ablation ensures a high bond quality. The coefficients of thermal expansion (CTE) are $8 \times 10^{-6}$ and $9 \times 10^{-6} \mathrm{~K}^{-1}$ for $a$ and $c$ axes of sapphire, respectively, and $17.2 \times 10^{-6} \mathrm{~K}^{-1}$ for stainless steel [27]. However, the intermixing of sapphire and steel in the interface could contribute to reducing the difference in CTEs, which is a key requirement in welded systems.

In order to study the influence of the laser welding in the electrical properties of the stainless steel foils, the electrical resistance through the bonded lines was measured. Figures 7 and 8 display the four needles configuration in one of the reference stripes and in one of the bonded stripes with the sapphire, respectively. The slope of the I-V linear regression fits (not shown) corresponds to the electrical resistance. The results are displayed in Table 2. The resistance values of the bonded stainless steel stripes are slightly higher than that of the reference stripes (without laser process), $303.78 \mathrm{~m} \Omega$ against $291.14 \mathrm{~m} \Omega$, respectively. This indicates a small loss of electrical conductivity of the steel stripe after the laser welding process. However, this increment in the resistivity in comparison with the pure stainless steel is acceptable. Moreover, the specific resistances were also calculated according to the volume of the steel stripes, in order to compare it with data available in the literature. The specific resistance of the reference steel, $7.28 \Omega \mathrm{m}$, greatly agrees with the data available for AISI $304,7.2 \Omega \mathrm{m}$ [27]. The integrity of the steel composition (Table 1) in the interface after re-solidification leads to 

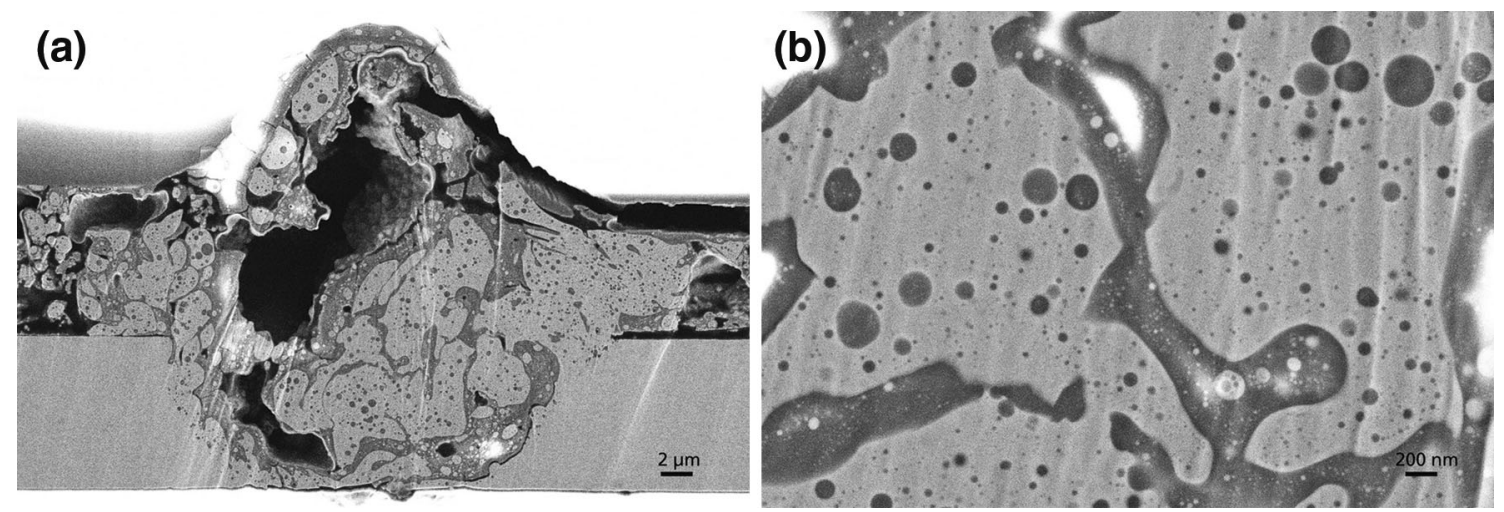

Fig. 5 SEM micrograph employing a secondary electron detector of the cross section of the stainless steel/sapphire interface a and detailed view of the craters $b$

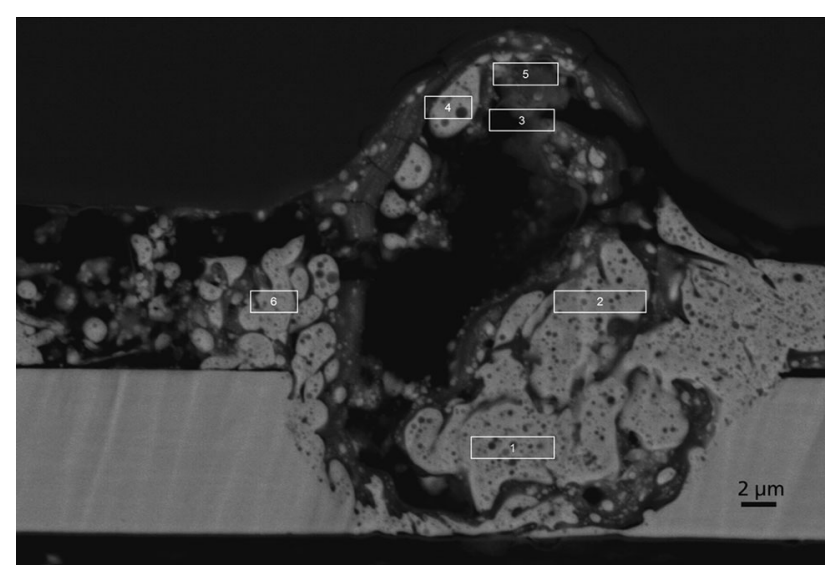

Fig. 6 SEM micrograph employing an angle-selective backscattered electron detector (AsB) of the cross section of the stainless steel/ sapphire interface. White boxes indicate the areas for the EDXS analysis

Table 1 Element concentration (at.\%) from the EDXS analysis at different areas of the interface in Fig. 6 and that of pure stainless steel

\begin{tabular}{llrll}
\hline Area & Al-K & Cr-K & Fe-K & Fe/Al ratio \\
\hline 1 & 16.69 & 16.46 & 66.85 & $4: 1$ \\
2 & 58.79 & 10.06 & 31.15 & $1: 2$ \\
3 & 55.06 & 9.69 & 35.25 & $1: 1.5$ \\
4 & 40.76 & 14.35 & 44.89 & $1: 1$ \\
5 & 26.33 & 15.01 & 58.66 & $2: 1$ \\
6 & 25.30 & 15.72 & 58.98 & $2: 1$ \\
Stainless steel & & 18.13 & 66.55 &
\end{tabular}

The Fe/Al ratio in the different areas is also included

slight electrical conductivity changes upon laser welding. Since the laser process is much localized and the heataffected zone is in the micrometer range (Fig. 5), the laser process only slightly affects the conductivity of the stainless steel. In Ref. [3], it was reported a stainless steel substrate laser-welded with a sensor chip of the same

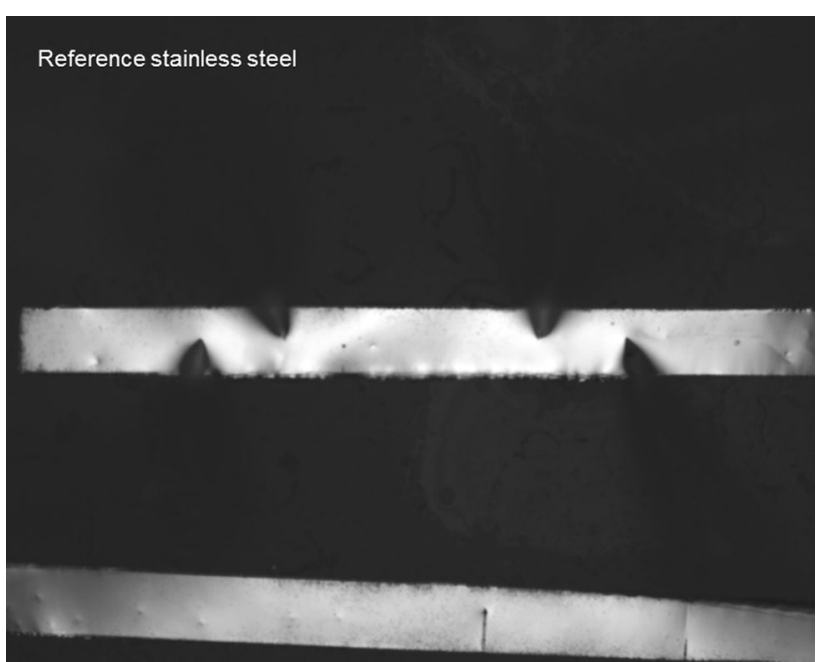

Fig. 7 Micrograph of the 4-needle setup in one reference stainless steel stripe. The width of the stripes is $800 \mu \mathrm{m}$

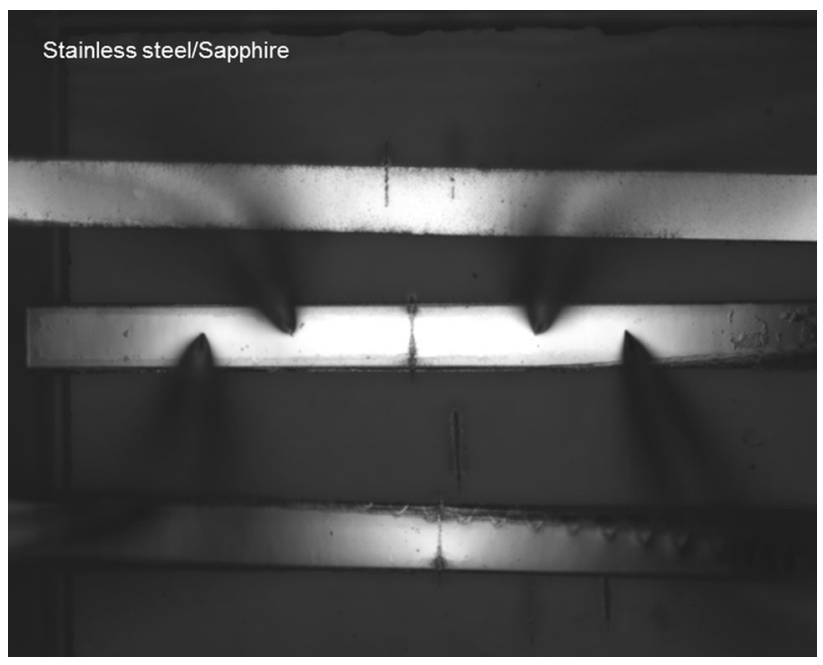

Fig. 8 Micrograph of the 4-needle setup in one of the laser-welded stainless steel stripes with sapphire. The width of the stripes is $800 \mu \mathrm{m}$ 
Table 2 Resistance values of the reference stripes and those welded with sapphire

\begin{tabular}{lll}
\hline Sample & $\begin{array}{l}\text { Resistance } \\
(\mathrm{m} \Omega)\end{array}$ & $\begin{array}{l}\text { Specific resistance } \\
\left(10^{-7} \Omega \mathrm{m}\right)\end{array}$ \\
\hline Reference & 291.17 & 7.28 \\
Welded foil 1 & 318.94 & 7.97 \\
Welded foil 2 & 297.29 & 7.43 \\
Welded foil 3 & 299.91 & 7.50 \\
Welded foil 4 & 298.98 & 7.47 \\
Average value & 303.78 & 7.59 \\
\hline
\end{tabular}

The values are the average of three measurements in each stripe

material. The performed electrical measurements showed a six times higher electrical conductance compared with the conventional epoxy adhesive, proving clear advantages of the laser welding approach.

\section{Conclusions}

Stainless steel foils as thin as $10 \mu \mathrm{m}$ were welded to sapphire substrates by irradiation with a ns pulsed laser at $532 \mathrm{~nm}$ employing an optimized setup and laser parameters. The microstructure analysis of the bond interface indicates that the sapphire and the steel foil were ablated under the laser irradiation and a mixture of both materials constitutes the interface, ensuring a good intermixing of the bonded materials. The electrical conductivity between the bonded area decreases only slightly in comparison with the non-processed steel foils. This work opens a new route to join metallic foils to transparent materials.

Acknowledgments The authors acknowledge the financial support of Fraunhofer Internal Programs under Grant No. Attract 692280.

\section{References}

[1] J. Ma, Displays 37, 2 (2015)

[2] N. Marsi, B.Y. Majlis, A.A. Hamzah, F. Mohd-Yasin, Microsyst. Technol. 21, 319 (2014)

[3] X. Chen, D. Brox, B. Assadsangabi, M.S. Mohamed Ali, K. Takahata, in Abstracts of Transducers 2015-18th International Conference on Solid-State Sensors, Actuators and Microsystems Alaska, USA
[4] C.H. Cheng, A.S. Yang, C.J. Lin, W.J. Huang, Microsyst. Technol. (2015). doi:10.1007/s00542-015-2769-z)

[5] T. Suzuki, I. Kanno, J.J. Loverich, H. Kotera, K. Wasa, Sens. Actuators A 125, 382 (2006)

[6] Z. Xie, L.-S. Hung, F. Zhu, Chem. Phys. Lett. 381, 691 (2003)

[7] X.M. Yu, J.X. Sun, X.L. Zhu, M. Wong, H.S. Kwo, SID Symp. Digest Tech. Pap. 39, 2064 (2008)

[8] W.-J. Lee, D.-H. Cho, J.-H. Wi, W.S. Han, J. Kim, Y.-D. Chung, Mater. Chem. Phys. 147, 783 (2014)

[9] E.R. Dobrovinskaya, L.A. Litvinov, V.V. Pishchik, Sapphire: Material, Manufacturing, Applications (Springer Science \& Business Media, New York, USA, 2009)

[10] L. Qi, K. Nishii, M. Yasui, H. Aoki, Y. Namba, Opt. Laser. Eng. 48, 1000 (2010)

[11] M. Pollnau, in Abstracts of IEEE LEOS Annual Meeting 2008 California, USA, pp. 455

[12] T. Zaharinie, R. Moshwan, F. Yusof, M. Hamdi, T. Ariga, Mater. Design 54, 375 (2014)

[13] H. Ning, F. Huang, J. Ma, Z. Geng, Z. Han, Rare Metal Mater. Eng. 32, 236 (2003)

[14] C. Varmazis, R. Viswanathan, R. Caton, Rev. Sci. Instrum. 49, 549 (1978)

[15] A. de Pablos-Martín, S. Tismer, T. Höche, J. Mater. Sci. Technol. 31, 484 (2015)

[16] A. de Pablos-Martín, M. Ebert, C. Patzig, M. Krause, M. Dyrba, P. Miclea, M. Lorenz, M. Grundmann, T. Höche, Microsyst. Technol. 21, 1035 (2014)

[17] A. de Pablos-Martin, S. Tismer, G. Benndorf, M. Mittag, M. Lorenz, M. Grundmann, T. Höche, Opt. Laser Technol. 81, 153 (2016)

[18] A. de Pablos-Martín, S. Tismer, F. Naumann, M. Krause, M. Lorenz, M. Grundmann, T. Höche, Microsyst. Technol. 22, 207 (2016)

[19] F.I. Hussein, E. Akman, B. Genc Oztoprak, M. Gunes, O. Gundogdu, E. Kacar, K.I. Hajim, A. Demir, Opt. Laser Technol. 49, 143 (2013)

[20] R.M. Carter, J. Chen, J.D. Shephard, R.R. Thomson, D.P. Hand, Appl. Opt. 53, 4233 (2014)

[21] T. Scholz, K. Dickmann, A. Ostendorf, H. Uphoff, M. Michalewicz, J. Laser Appl. 27, 032001 (2015)

[22] D. Bergström, J. Powell, A.F.H. Kaplan, Appl. Surf. Sci. 253, 5017 (2007)

[23] K. Nagayama, Y. Utsunomiya, T. Kajiwara, T. Nishiyama, in Pulse Laser Ablation by Reflection of Laser Pulse at Interface of Transparent Materials, in Lasers-Applications in Science and Industry, ed. by Krzysztof Jakubczak (2011)

[24] M.W. Greenaway, W.G. Proud, J.E. Field, S.G. Goveas, Rev. Sci. Instrum. 73, 2185 (2002)

[25] S. Chen, J. Huang, J. Xia, H. Zhang, X. Zhao, Metall. Mater. Trans. A 44, 3690 (2013)

[26] M. Yoshitake, M. Tosa, K. Yoshihara, Thin Solid Films 172, 35 (1989)

[27] J. Mangas-Murillo, E.M. Cuerda-Correa, J.R. Domínguez, A. Macías-García, E. Bernalte, J. Alloys Compd. 577, 360 (2013) 